\title{
Test Simulations of Temperature Screening Effect by using Kinetic Monte Carlo Model ${ }^{*}$
}

\author{
Yuki HOMMA ${ }^{1,2)}$, Akiyoshi HATAYAMA ${ }^{1)}$, Yu SAWADA ${ }^{1)}$ and Shohei YAMOTO ${ }^{1)}$ \\ ${ }^{1)}$ Graduate School of Science and Technology, Keio University, 3-14-1 Hiyoshi, Kohoku-ku, Yokohama 223-8522, Japan \\ ${ }^{2)}$ Research Fellow of Japan Society for the Promotion of Science
}

(Received 25 January 2014 / Accepted 11 May 2014)

\begin{abstract}
Temperature Screening Effect (TSE) on the impurity transport in fusion plasmas has been studied by a kinetic Monte Carlo simulation model [Y. Homma and A. Hatayama, J. Comput. Phys. 250, 206 (2013)]. The TSE drift is induced by the background-plasma tempetature gradient across the magnetic field, and is dependent on various parameters of the impurity species as well as on the background-plasma condition. A series of test simulations has been performed in wide range of parameter space. It has been confirmed that the parametric dependences of the TSE drift are correctly reproduced by our kinetic model.
\end{abstract}

(c) 2014 The Japan Society of Plasma Science and Nuclear Fusion Research

Keywords: temperature screening effect, thermal force, perpendicular temperature gradient, numerical model, parametric dependences

DOI: 10.1585/pfr.9.1403095

\section{Introduction}

Understanding of impurity behaviors in the ScrapeOff Layer (SOL) and the divertor region is one of the most important issues for developing fusion plasma devices, because of their strong influences (both negative and positive) on plasmas such as the radiation cooling.

Many impurity transport simulation codes using the test-particle scheme have been actively developed so far, e.g. DIVIMP [1], DORIS [2], IMPMC [3], ASCOT [4], and IMPGYRO [5]. In these codes, collisional effects are applied by the various Monte Carlo methods.

We have been developing a new kinetic Monte Carlo model in order to more correctly simulate the thermal force acting on test impurity ions in magnetized plasmas [6-8]. The background-plasma temperature gradient $\nabla_{\|} T_{b}$ parallel to the magnetic field $\boldsymbol{B}$ induces the thermal force parallel to $\boldsymbol{B}$, while its gradient $\nabla_{\perp} T_{b}$ perpendicular to $\boldsymbol{B}$ induces the thermal force both perpendicular to $\boldsymbol{B}$ and $\nabla_{\perp} T_{b}$ $[9,10]$. In our model, Coulomb collision is simulated by the Monte Carlo binary-collision method [11], and the velocity distribution of background plasma ions is modeled by a distorted Maxwellian. The distortion caused by the temperature gradient is given with an analytic formula. At first we took into account of only the effect of $\nabla_{\|} T_{b}[6,7]$. Afterwards we introduced both effects of $\nabla_{\|} T_{b}$ and $\nabla_{\perp} T_{b}$, with the full orbit-following model including the gyration motion. It has been shown that our model precisely simulates the perpendicular thermal force in addition to the parallel thermal force [8].

The perpendicular thermal force which is both perpen-

author's e-mail: yuki.homma@ppl.appi.keio.ac.jp

*) This article is based on the invited talk at the 30th JSPF Annual Meeting (2013, Tokyo). dicular to $\nabla_{\perp} T_{b}$ and to $\boldsymbol{B}$, is called hereafter the diamagnetic thermal force. The diamagnetic thermal force drives guiding-center drift of impurities. As shown in Fig. 1, the impurities are transported across the $\boldsymbol{B}$-field, toward the opposite direction to the temperature gradient $\nabla_{\perp} T_{b}$. This

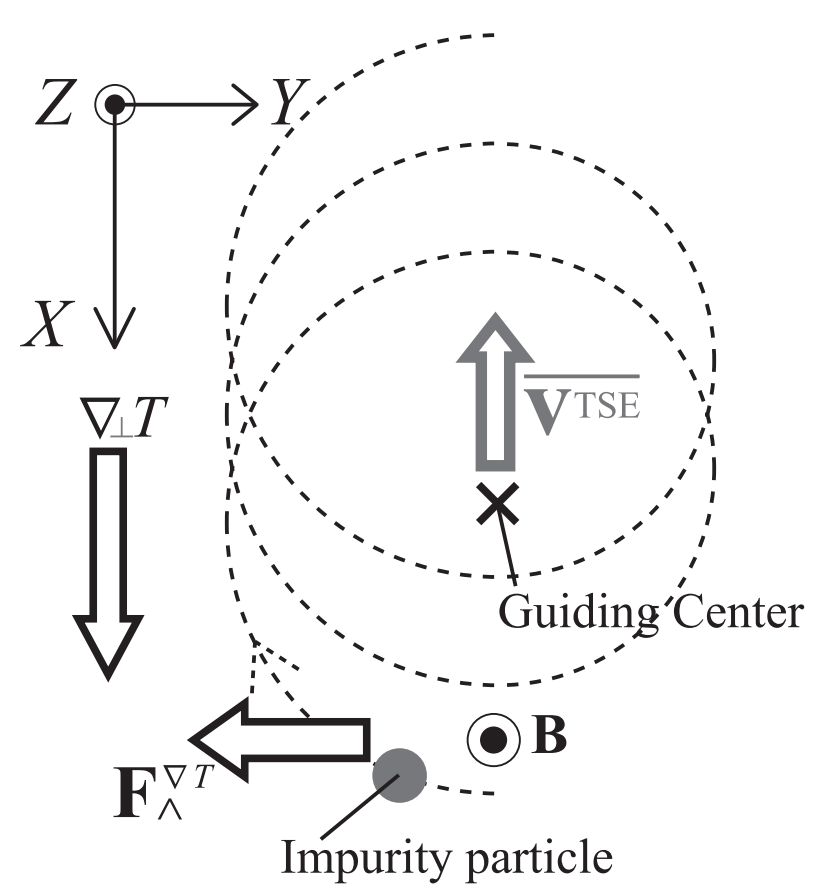

Fig. 1 Guiding center drift of Temperature Screening Effect (TSE). The diamagnetic thermal force $\boldsymbol{F}_{\wedge}^{\nabla T}$ acts on the impurity particle perpendicularly both to the temperature gradient $\nabla_{\perp} T_{b}$ and the magnetic field $\boldsymbol{B}$. The consequent TSE drift drives the impurity toward the opposite direction of the gradient $\left(-\nabla_{\perp} T_{b}\right)$. 
transport process is called the temperature screening effect (TSE) [10]. It has been recently pointed out in Ref. [8] that the TSE may have non-negligible effect on impurity transport in the SOL/Divertor region in fusion plasmas where the relatively strong perpendicular temperature gradient exists. The TSE has not been taken into account in any existing impurity transport simulation codes with testparticle scheme.

The diamagnetic thermal force and the velocity of TSE guiding center drift are dependent on various parameters of the impurity species as well as on the background plasma condition. In Ref. [8], however, it has not been examined whether our model is able to reproduce such parametric dependences of TSE drift.

In the present study, we have checked the dependences of TSE drift on various parameters; the impurity charge state and mass, the background-plasma density, temperature, magnitude of temperature gradient, and the magnetic field strength.

In Sec. 2, we will briefly review our Monte Carlo model of thermal force and the theoretical expressions of TSE drift including its parametric dependences. In Sec. 3, a series of test simulations will be performed to confirm whether our numerical model is able to reproduce such parametric dependences of TSE drift. Summary will be given in Sec. 4.

\section{Kinetic Monte Carlo Model of Thermal Force and TSE Drift}

\subsection{Kinetic Monte Carlo model to simulate thermal force acting on test impurity ions}

Our numerical simulation model [6-8] is able to correctly simulate the thermal force acting on individual test impurities in magnetized plasma, taking into account the background temperature gradient both parallel and perpendicular to the magnetic field.

Basic characteristics of the model are summarized as follows: (1) Full orbit of individual test impurity ion is exactly solved by the equation of motion. (2) Coulomb collisions between impurities and background plasma ions are simulated by the binary-collision method [11]. (3) In our binary-collision algorithm, the background ion velocities are randomly sampled from a distorted Maxwellian distribution which takes into account the spatial gradient of background plasma temperature (details are described in Ref. [8]).

The step (3) above is an original point of our model. The use of distorted Maxwellian makes it possible to simulate the thermal force. Most conventional models for kinetic impurity transport simulation assume the velocity distribution of background plasma simply as shifted Maxwellian.

\subsection{TSE drift and its parameter depen- dences}

As described in Refs. [8, 9], perpendicular temperature gradient $\nabla_{\perp} T_{b}$ in a strongly magnetized and collisional background plasma, generates the diamagnetic thermal force $\boldsymbol{F}_{\wedge}^{\nabla T}$ acting on impurity ions moving in this plasma. The diamagnetic thermal force $\boldsymbol{F}_{\wedge}^{\nabla T}$ drives the TSE drift of impurities, as illustrated schematically in Fig. 1. The drift velocity of TSE has already been evaluated from the fluid equations in Ref. [10]:

$$
\boldsymbol{v}_{a}^{\mathrm{TSE}}=-\frac{3}{2} \frac{m_{b}}{q_{a} q_{b} B^{2}} \frac{n_{b}}{n_{a} \tau_{b a}} \cdot \nabla_{\perp} T_{b},
$$

where the impurity particle's electric charge and density are $q_{a}$ and $n_{a}$, respectively. The background plasma ion's mass, electric charge, density, and temperature (energy unit) are, respectively, $m_{b}, q_{b}, n_{b}$, and $T_{b}$. The symbol $\tau_{b a}$ denotes the collision time between the background plasma ion and the impurity. The magnetic field strength is $B:=|\boldsymbol{B}|$. The perpendicular temperature gradient is defined with respect to the direction of magnetic field as $\nabla_{\perp} T_{b}:=\boldsymbol{B} \times\left(\nabla T_{b} \times \boldsymbol{B}\right) / B^{2}$.

As discussed in Ref. [8], there are two candidates for the collision time $\tau_{b a}$. One is the definition given by Braginskii [9],

$$
\tau_{b a}^{\mathrm{B}}:=\frac{12 \pi^{3 / 2}}{\sqrt{2}} \frac{\epsilon_{0}^{2} \sqrt{m_{b}} T_{b}^{3 / 2}}{n_{a} q_{a}^{2} q_{b}^{2} \ln \Lambda},
$$

where $\epsilon_{0}$ is the permittivity of vacuum and $\ln \Lambda$ is the Coulomb logarithm. The Braginskii collision time $\tau_{b a}^{\mathrm{B}}$ assumes the condition that $n_{a} q_{a}^{2} /\left(n_{b} q_{b}^{2}\right)=1$. The other definition of collision time is given by Trubnikov [12],

$$
\tau_{b a}^{\mathrm{T}}:=8 \sqrt{2} \pi \frac{\epsilon_{0}^{2} \sqrt{m_{b}} T_{b}^{3 / 2}}{n_{a} q_{a}^{2} q_{b}^{2} \ln \Lambda},
$$

under the condition that $m_{a} \gg m_{b}$.

In the present study, we use the both collision times $\tau_{b a}^{\mathrm{B}}$ and $\tau_{b a}^{\mathrm{T}}$ for estimating the TSE drift velocity $\boldsymbol{v}_{a}^{\mathrm{TSE}}$ in Eq. (1), because the both characteristic times are only slightly different in their numerical factor, $\tau_{b a}^{\mathrm{B}}: \tau_{b a}^{\mathrm{T}}=1$ : 0.75. The parameter dependences of $\boldsymbol{v}_{a}^{\mathrm{TSE}}$ are not affected by the choice of $\tau_{b a}^{\mathrm{B}}$ and $\tau_{b a}^{\mathrm{T}}$.

From Eq. (1), the TSE drift has the parameter dependences such as,

$$
\left|\boldsymbol{v}_{a}^{\mathrm{TSE}}\right| \propto \sqrt{m_{b}} \frac{q_{a} q_{b} n_{b}\left|\nabla_{\perp} T_{b}\right|}{B^{2} T^{3 / 2}}
$$

In the following sections, the TSE drift simulated by our model will be compared with the theoretical values of Eqs. (1-4).

\section{Test Simulations}

The parametric dependences of TSE drift on (1) the impurity charge state $Z$, (2) the impurity mass number $M$, (3) the background plasma density $n_{b}$, (4) the background 
Table 1 Reference condition for test simulations.

\begin{tabular}{lr}
\hline \hline Test impurity species & $\mathrm{W}^{3+}$ with charge state $Z=+3$ \\
Initial position of impurities, $\boldsymbol{r}_{a, i}(t=0)$ & $\mathbf{0}$ \\
Initial velocity of impurities, $\boldsymbol{v}_{a, i}(t=0)$ & Maxwellian with $T_{a}=50 \mathrm{eV}$ \\
No. of test particles, $N$ & $10^{4}$ \\
\hline Background plasma ion species & $\mathrm{H}^{+}$ \\
Background plasma ion density, $n_{b}$ & $10^{20} \mathrm{~m}^{-3}$ \\
Background flow velocity, $\overline{\boldsymbol{v}_{b}}$ & $\mathbf{0 ~ m} / \mathrm{s}$ \\
Temperature at the origin, $T_{b}(\mathbf{0})$ & $50 \mathrm{eV}$ \\
Temperature gradient, $\nabla_{\perp} T_{b}$ & $300 \mathrm{eV} / \mathrm{m} \boldsymbol{e}_{X}$ \\
\hline Magnetic field $\boldsymbol{B}$ & $1 \mathrm{~T} \boldsymbol{e}_{Z}$ \\
Coulomb logarithm, $\ln \Lambda$ & 15 \\
\hline Simulation time step, $\Delta t$ & $2.03 \times 10^{-10} \mathrm{~s}$ \\
Gyration period of test particle, $\Omega_{a}^{-1}$ & $6.40 \times 10^{-7} \mathrm{~s}$ \\
Total calculated time, $t$ & $0 \leq \Omega_{a} t \leq 650$ \\
\hline \hline
\end{tabular}

plasma temperature $T_{b}$, (5) the magnitude of perpendicular temperature gradient $\left|\nabla_{\perp} T_{b}\right|$, and (6) the magnetic field strength $B$, are examined in the following test simulations. The values of these parameters are chosen from the ranges which are typical for SOL/Divertor region of fusion plasmas.

At first, we present a reference condition. Next, the parametric dependences of TSE are simulated by changing only one parameter at a time.

\subsection{Reference condition}

As the reference, we use the condition of Case (II-2) in Ref. [8], which we have summarized in Table 1. The test simulation geometry is shown in Fig. 1. We use the Cartesian coordinates $(X, Y, Z)$. The unit vectors along each axis are expressed, respectively, as $\boldsymbol{e}_{X}, \boldsymbol{e}_{Y}$, and $\boldsymbol{e}_{Z}$. The magnetic field is $\boldsymbol{B}=1 \mathrm{~T} \boldsymbol{e}_{Z}$. The test impurity species is tungsten ion $\mathrm{W}^{3+}$ (mass $m_{a}$, electric charge $q_{a}$ ). The background plasma ion is hydrogen ion $\mathrm{H}^{+}$(mass $m_{b}$, electric charge $q_{b}$ ). Electrons are not considered. The plasma temperature at the origin is $T_{b}(\mathbf{0})=50 \mathrm{eV}$, and its spatial gradient is taken perpendicularly as $\nabla_{\perp} T_{b}=300 \mathrm{eV} / \mathrm{m} \boldsymbol{e}_{X}$. The background plasma ion density is $n_{b}=10^{20} \mathrm{~m}^{-3}$, and the background flow velocity $\overline{\boldsymbol{v}_{b}}$ is set to $\mathbf{0}$.

The simulation time step is appropriately decided as instructed in Ref. [8].

In the simulations, test impurity particles (the total number $N=10^{4}$ ) start moving from the coordinate origin. Initial velocity of each test particle is randomly chosen from the Maxwellian distribution with average energy of $T_{a}=50 \mathrm{eV}\left(=T_{b}\left(\boldsymbol{r}_{a}=\mathbf{0}\right)\right)$. Then we solve the trajectories of each test particle for a period of time long enough for the impurities to gyrate for many times $\left(0<\Omega_{a} t<650\right.$, where the Larmor gyro-frequency of impurity is defined by $\left.\Omega_{a}:=q_{a}|\boldsymbol{B}| / m_{a}\right)$, and we look into their TSE drift caused by thermal force.

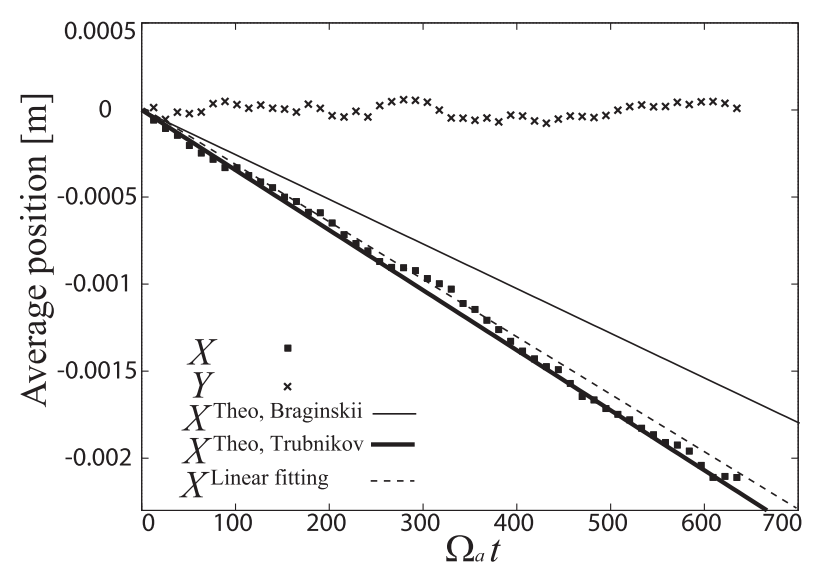

Fig. 2 Simulated results of the reference case. Closed squares and cross marks represent the time evolution of averaged $X$-position $\bar{X}(t)$ and the $Y$-position $\bar{Y}(t)$ of the impurities. The dashed line shows a linear approximation of $\bar{X}(t)$. The theoretical values $v_{a}^{\mathrm{TSE}}\left(\tau_{b a}=\tau_{b a}^{\mathrm{B}}\right)$ and $v_{a}^{\mathrm{TSE}}\left(\tau_{b a}=\tau_{b a}^{\mathrm{T}}\right)$, obtained with the Braginskii and Trubnikov collision time, are also presented by the thin/bold solid line, respectively.

As a result of calculation, Fig. 2 shows the time evolution of the average position of test impurities $\overline{\boldsymbol{r}}(t)$, which is defined as

$$
\overline{\boldsymbol{r}}(t):=\sum_{i=1}^{N} \boldsymbol{r}_{a, i}(t) / N,
$$

where $\boldsymbol{r}_{a, i}(t)$ is the position of $i$-th test impurity $(i=1 \sim N)$ at the time $t$. The vertical axis means the traveled distance, the horizontal axis is the time normalized to $\Omega_{a}^{-1}$. Closed squares and cross marks represent the value of the $X$-component $\bar{X}(t)$ and the $Y$-component $\bar{Y}(t)$ of the average position $\overline{\boldsymbol{r}}(t)$. The theoretical values calculated from Eqs. (1-3) are presented by the thin/bold solid line, respectively, with the Braginskii and Trubnikov collision time de- 
fined in Eqs. (2, 3).

The test particles are apparently moving as a whole toward the $(-X)$-direction, i.e. to lower temperature region across the $\boldsymbol{B}$-field, while its averaged $Y$-position remains around 0 .

More quantitatively, the average speed along the $X$ axis $\overline{v_{X}}(t):=\mathrm{d} \bar{X}(t) / \mathrm{d} t$ has been evaluated by using the least squares method. The simulated results have been approximated as linear function as shown in Fig. 2 by the dashed line, and the obtained value of $\overline{v_{X}}(t) \approx-5.32 \mathrm{~m} / \mathrm{s}$ agrees with the theoretical value of TSE $\operatorname{drift} v_{a}^{\mathrm{TSE}}\left(\tau_{b a}=\tau_{b a}^{\mathrm{T}}\right)=$ $-5.39 \mathrm{~m} / \mathrm{s}$.

The TSE drift of impurity is correctly simulated in the reference case.

\subsection{Dependence on impurity charge state}

From Eq. (4), the TSE drift velocity is proportional to the impurity charge state $Z$, which is defined by $Z:=q_{a} / q_{e}$ with the elementary charge $q_{e}:=1.602 \times 10^{-19} \mathrm{C}$.

We have changed the value of $Z$ from 1 to 15 . Other parameters remain unchanged from the reference condition (Table 1), and the test simulations have been performed in the same manner as the reference case. The simulated TSE drift velocities of test impurities have been evaluated by the least squares method as in the reference case. These results are plotted in Fig. 3 by closed square marks, according to the number of charge state $Z$. The result of the reference condition is marked as an emphasized square. The theoretical values $v_{a}^{\mathrm{TSE}}\left(\tau_{b a}=\tau_{b a}^{\mathrm{B}}\right)$ and $v_{a}^{\mathrm{TSE}}\left(\tau_{b a}=\tau_{b a}^{\mathrm{T}}\right)$ are also presented in Fig. 3.

The calculated drift speeds in Fig. 3 are almost linearly increasing with the impurity charge state $Z$. It is consistent with the expected tendency $v^{\mathrm{TSE}} \propto Z$ in Eq. (4).

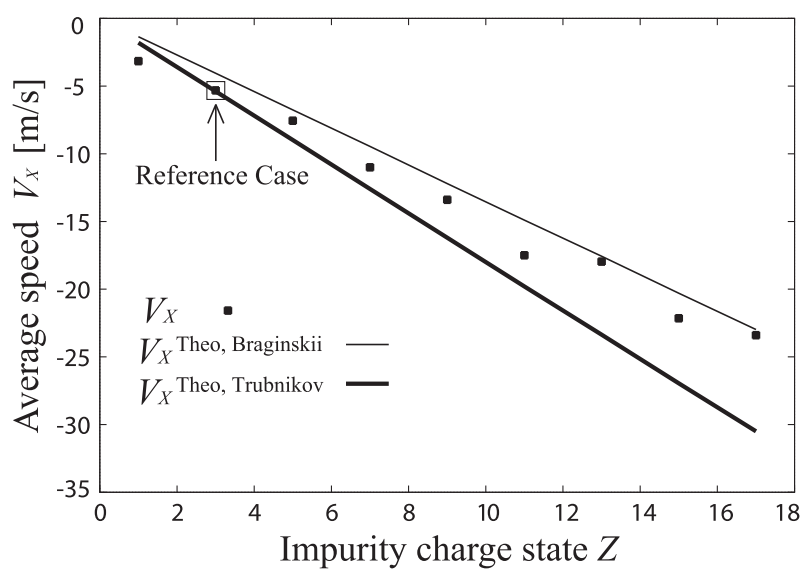

Fig. 3 Dependence of TSE on impurity charge state. The simulated TSE drift velocity of test impurities is plotted by closed square marks, according to the number of charge state $Z$. The result of the reference condition is marked as an emphasized square. The theoretical values $v_{a}^{\mathrm{TSE}}\left(\tau_{b a}=\right.$ $\left.\tau_{b a}^{\mathrm{B}}\right)$ and $v_{a}^{\mathrm{TSE}}\left(\tau_{b a}=\tau_{b a}^{\mathrm{T}}\right)$ are also presented, respectively, by the thin/bold solid line.
The slope of simulated data points tends to be larger compared with the theoretical prediction by Braginskii, while smaller than that by Trubnikov. This discrepancy from the fluid approximations Eqs. (1-3) may possibly be due to the kinetic effect, especially finite Larmor radius (FLR) effect which has not been taken into account in the fluid modeling.

In order to investigate the FLR effect, we have performed test simulations by artificially supposing a very ideal background plasma where temperature gradient exists but its temperature profile is uniform. In such ideal condition, the impurities receive the thermal force but they always feel the same background temperature. The FLR effect is thus suppressed. The resultant drift speed $v^{\mathrm{TSE}}$ without FLR effect and its $Z$-dependence agreed well with the theoretical prediction by Braginskii. More systematic study on FLR effects will be performed in the future.

\subsection{Dependence on impurity mass number}

It is expected from Eq. (4) that the TSE drift is affected neither by the impurity mass $m_{a}$ nor by its mass number $M:=m_{a} / m_{p}\left(m_{p}:=1.672 \times 10^{-27} \mathrm{~kg}\right.$ is the mass of proton).

Here we have changed the mass number of impurity $M$ from 4 to 190, covering from the helium to the tungsten. Other parameters are the same as the reference condition in Table 1. The test simulations have been performed as in the previous sections.

Figure 4 shows the simulated TSE drift velocity and their theoretical values, for each mass number of impurity species. It is seen that the TSE drift is almost insensitive to the impurity mass number when $M \geq 10$. We have confirmed that the mass independence of TSE drift can be cor-

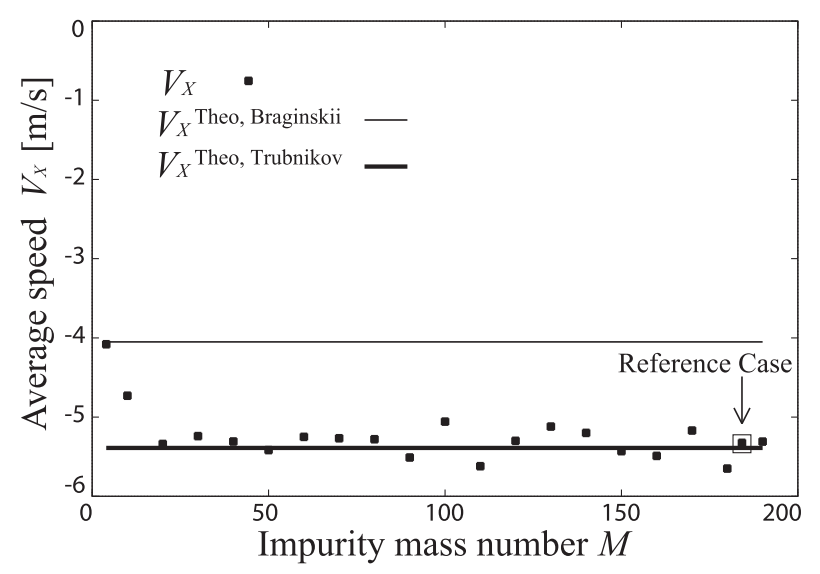

Fig. 4 Dependence of TSE on impurity mass number. The simulated TSE drift velocity of test impurities is plotted by closed square marks, according to the mass number of impurity $M$. The result of the reference condition is marked as an emphasized square. The theoretical values $v_{a}^{\mathrm{TSE}}\left(\tau_{b a}=\tau_{b a}^{\mathrm{B}}\right)$ and $v_{a}^{\mathrm{TSE}}\left(\tau_{b a}=\tau_{b a}^{\mathrm{T}}\right)$ are also presented, respectively, by the thin/bold solid line. 
rectly simulated by our model for a large part of impurity mass number $M$.

For lower mass number $M \leq 10$, the TSE drift velocity decreases and seems not to be independent of $M$ any more. This result is understood from the mass dependence of the thermal force, which is omitted both in the fluid approximation Eq. (2) by Braginskii and in the basic relaxation time Eq. (3) by Trubnikov. The kinetic formula of the thermal force [8] is given as

$$
\begin{aligned}
\boldsymbol{F}^{\nabla T}= & -\frac{\ln \Lambda}{10 \pi \sqrt{\pi}}\left(\frac{q_{a} q_{b}}{\epsilon_{0}}\right)^{2} \sqrt{\frac{m_{b}}{2 T_{b}}} \frac{1}{T_{b}^{2}}\left(1+\frac{m_{b}}{m_{a}}\right) \\
& \times \exp \left(-\tilde{v}_{a}^{2}\right) \cdot\left[\boldsymbol{q}-2\left(\boldsymbol{q} \cdot \tilde{\boldsymbol{v}}_{a}\right) \tilde{\boldsymbol{v}}_{a}\right],
\end{aligned}
$$

with the heat flux vector of background plasma $\boldsymbol{q}$ and the normalized impurity velocity $\tilde{\boldsymbol{v}}_{a}:=\sqrt{m_{b} /\left(2 T_{b}\right)}\left(\boldsymbol{v}_{a}-\overline{\boldsymbol{v}_{b}}\right)$. Since the impurity velocity $\boldsymbol{v}_{a}$ is initially randomly chosen in such a way that $m_{a}\left\langle v_{a}^{2}\right\rangle / 2=(3 / 2) T_{b}$, the following estimation would stand:

$$
\begin{aligned}
& \tilde{v}_{a}^{2}=\frac{m_{b}}{2 T_{b}} v_{a}^{2} \approx \frac{3}{2} \frac{m_{b}}{m_{a}}, \\
&\left|\boldsymbol{F}^{\nabla T}\right| \propto\left(1+\frac{m_{b}}{m_{a}}\right) \exp \left(-\tilde{v}_{a}^{2}\right) \cdot\left[\boldsymbol{q}-2\left(\boldsymbol{q} \cdot \tilde{\boldsymbol{v}}_{a}\right) \tilde{\boldsymbol{v}}_{a}\right] \\
& \quad \approx\left(1+\frac{m_{b}}{m_{a}}\right) \exp \left(-\frac{3}{2} \frac{m_{b}}{m_{a}}\right) .
\end{aligned}
$$

When we take the limit of heavy impurity $m_{a} \gg m_{b}$, the diamagnetic thermal force and the TSE drift velocity become independent of $m_{a}$, as expected by the fluid approximation in Eq. (4). On the other hand, when the impurity mass gets smaller as much as about $m_{a} / m_{b} \approx 1$, the diamagnetic thermal force is weakened by the coefficient $\left(1+m_{b} / m_{a}\right) \exp \left(-m_{b} / m_{a}\right)<1$ in Eq. (8). The TSE drift speed is reduced as well. The results for lower mass number $M \leq 10$ in Fig. 4 are understood from such mass dependence of the thermal force.

\subsection{Dependence on background ion density}

After checking the parameters relative to the impurity species, we consider the parameters characterizing the background plasma. The linear relation of TSE drift to the background plasma density ( $v^{\mathrm{TSE}} \propto n_{b}$ from Eq. (4)) is examined.

By changing the value of background density $n_{b}$ from $1 \times 10^{19} \mathrm{~m}^{-3}$ to $1 \times 10^{21} \mathrm{~m}^{-3}$, the same simulations as in the previous sections have been performed.

The simulated TSE drift velocity is presented in Fig. 5, with respect to the background density. The theoretical values of TSE are also shown. The results agree well with the theoretical values, and the linear relation to the background density $\left(v^{\mathrm{TSE}} \propto n_{b}\right)$ is reproduced by our numerical model.

\subsection{Dependence on background tempera- ture}

The TSE drift velocity is relatively strongly affected by the temperature of background plasma $T_{b}$, i.e. $v^{\mathrm{TSE}} \propto$ $T^{-3 / 2}$ from Eq. (4).

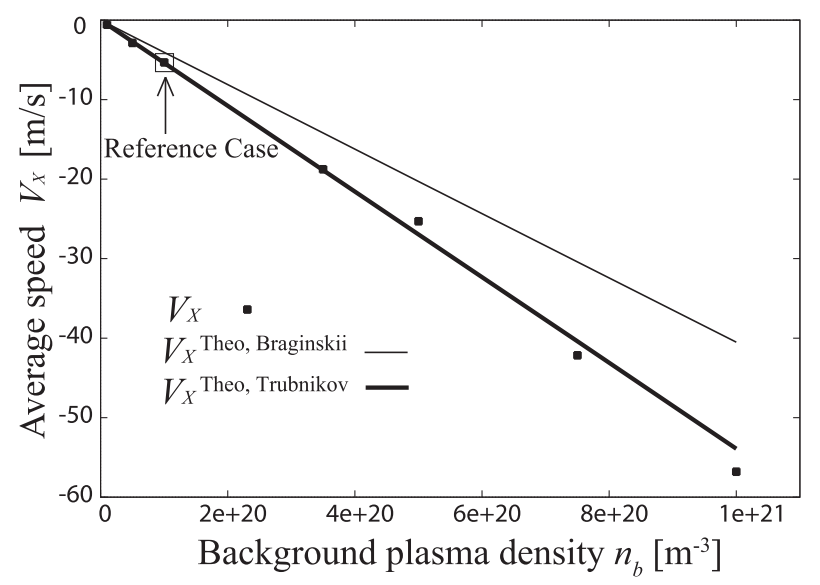

Fig. 5 Dependence of TSE on background plasma density. The simulated TSE drift velocity of test impurities is plotted by closed square marks, according to the background density $n_{b}$. The result of the reference condition is marked as an emphasized square. The theoretical values $v_{a}^{\mathrm{TSE}}\left(\tau_{b a}=\tau_{b a}^{\mathrm{B}}\right)$ and $v_{a}^{\mathrm{TSE}}\left(\tau_{b a}=\tau_{b a}^{\mathrm{T}}\right)$ are also presented, respectively, by the thin/bold solid line.

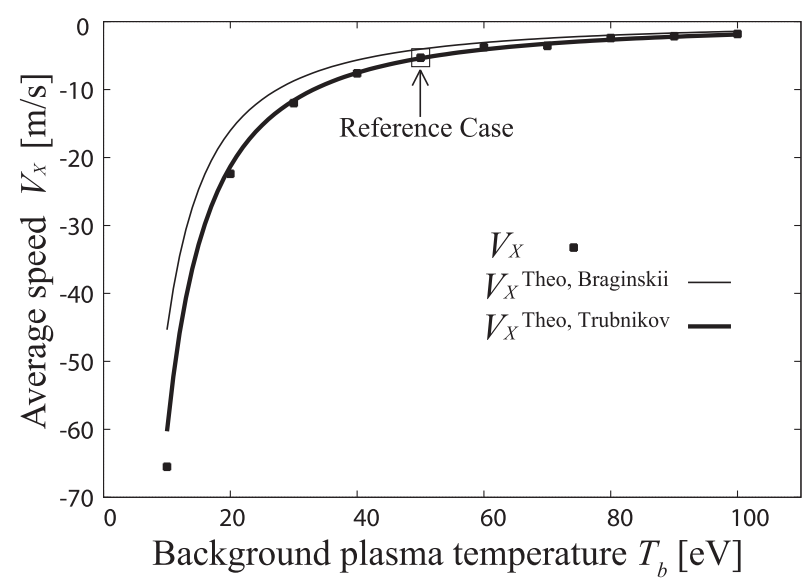

Fig. 6 Dependence of TSE on background plasma temperature. The simulated TSE drift velocity of test impurities is plotted by closed square marks, according to the background temperature $T_{b}$. The result of the reference condition is marked as an emphasized square. The theoretical values $v_{a}^{\mathrm{TSE}}\left(\tau_{b a}=\tau_{b a}^{\mathrm{B}}\right)$ and $v_{a}^{\mathrm{TSE}}\left(\tau_{b a}=\tau_{b a}^{\mathrm{T}}\right)$ are also presented, respectively, by the thin/bold solid line.

The value of background temperature has been varied from $10 \mathrm{eV}$ to $100 \mathrm{eV}$, and the test simulations have been performed. Figure 6 present the simulated results which show good agreement with the theoretical values. The temperature dependence of TSE drift $\left(v^{\mathrm{TSE}} \propto T^{-3 / 2}\right)$ is correctly reproduced by our model.

\subsection{Dependence on magnitude of perpendic- ular temperature gradient}

The TSE drift velocity is expected to be proportional to the magnitude of perpendicular temperature gradient, 


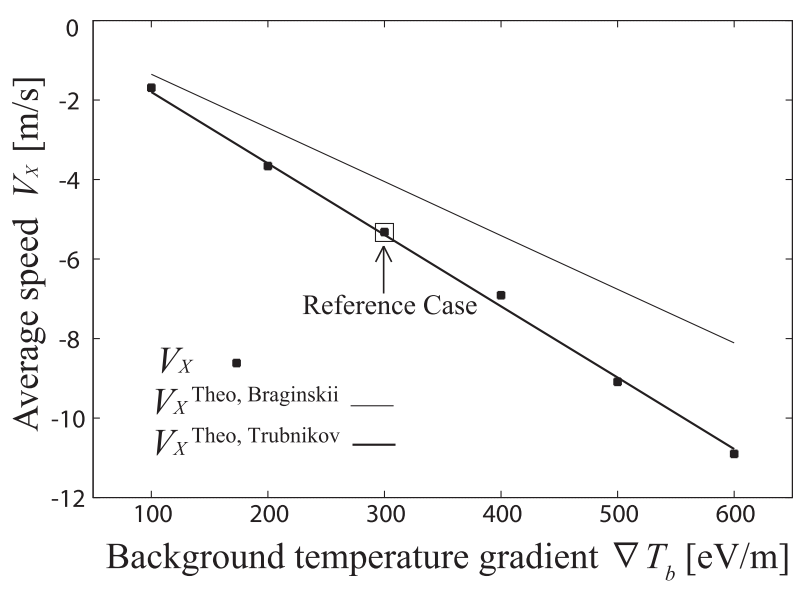

Fig. 7 Dependence of TSE on perpendicular temperature gradient. The simulated TSE drift velocity of test impurities is plotted by closed square marks, according to the magnitude of perpendicular temperature gradient, $\left|\nabla_{\perp} T_{b}\right|$. The result of the reference condition is marked as an emphasized square. The theoretical values $v_{a}^{\mathrm{TSE}}\left(\tau_{b a}=\tau_{b a}^{\mathrm{B}}\right)$ and $v_{a}^{\mathrm{TSE}}\left(\tau_{b a}=\tau_{b a}^{\mathrm{T}}\right)$ are also presented, respectively, by the thin/bold solid line.

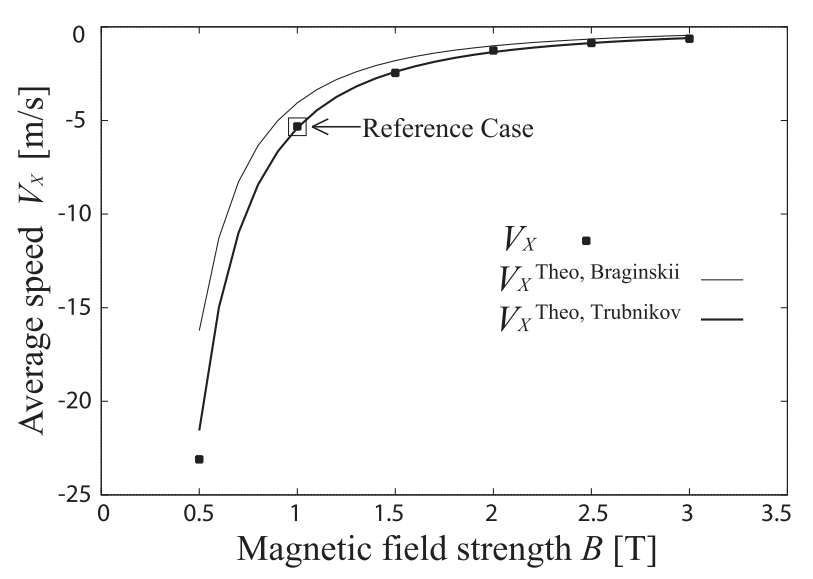

Fig. 8 Dependence of TSE on magnetic field strength. The simulated TSE drift velocity of test impurities is plotted by closed square marks, according to the magnetic field strength $B$. The result of the reference condition is marked as an emphasized square. The theoretical values $v_{a}^{\mathrm{TSE}}\left(\tau_{b a}=\tau_{b a}^{\mathrm{B}}\right)$ and $v_{a}^{\mathrm{TSE}}\left(\tau_{b a}=\tau_{b a}^{\mathrm{T}}\right)$ are also presented, respectively, by the thin/bold solid line.

$v^{\mathrm{TSE}} \propto\left|\nabla_{\perp} T_{b}\right|$. The value of $\left|\nabla_{\perp} T_{b}\right|$ has been varied from $100 \mathrm{eV} / \mathrm{m}$ to $600 \mathrm{eV} / \mathrm{m}$, and the test simulations have been performed.

Figure 7 shows good agreement between the simulated results of TSE drift velocity and their theoretical values. The linear dependence of TSE drift on the perpendicular temperature gradient is correctly reproduced by our model.

\subsection{Dependence on magnetic field strength}

The TSE drift velocity is strongly dependent on the magnetic field strength $\left(v^{\mathrm{TSE}} \propto B^{-2}\right)$. The test simulations have been performed by varying the magnitude of $\boldsymbol{B}$-field from $0.5 \mathrm{~T}$ to $3 \mathrm{~T}$.

Figure 8 presents the simulated results with respect to the value of $|\boldsymbol{B}|$. Good agreement between the simulation results and the theoretical values is clearly seen in the figure. The dependence of TSE drift on the $\boldsymbol{B}$-field strength is correctly taken into account by our model.

\section{Summary}

We have confirmed that our numerical model is able to simulate the TSE drift of test impurity ions caused by the diamagnetic thermal force, including the correct parametric dependences $\left|\boldsymbol{v}_{a}^{\mathrm{TSE}}\right| \propto \sqrt{m_{b}} q_{a} q_{b} n_{b}\left|\nabla_{\perp} T_{b}\right| /\left(B^{2} T^{3 / 2}\right)$.

Our model is useful for the kinetic transport simulation of impurity particles in fusion plasmas. The TSE drift of impurities have to be correctly simulated, especially in the SOL/Divertor region where the TSE could be enhanced by the following plasma conditions: (1) lower temperature, (2) steep temperature gradient, and (3) increased background density.

Our next goal is to implement this model into realistic simulation codes such as the IMPGYRO [5], in order to provide more precise kinetic impurity transport simulations which are indispensable for developing nuclear fusion devices.

\section{Acknowledgments}

This work was supported by Grant-in-Aid for JSPS (Japan Society for the Promotion of Science) Fellows, Grant Number 25-4952.

[1] P.C. Stangeby, C. Farrell, S. Hoskins et al., Nucl. Fusion 28, 1945 (1988).

[2] D. Reiser, D. Reiter and M.Z. Tokar', Nucl. Fusion 38, 165 (1998).

[3] K. Shimizu, H. Kubo, T. Takizuka et al., J. Nucl. Mater. 220-222, 410 (1995).

[4] J. Miettunen, T. Kurki-Suonio, T. Makkonen et al., Nucl. Fusion 52, 032001 (2012).

[5] A. Fukano, M. Noritake, K. Hoshino et al., J. Nucl. Mater. 363-365, 211 (2007).

[6] Y. Homma and A. Hatayama, J. Comput. Phys. 231, 3211 (2012).

[7] Y. Homma and A. Hatayama, Contrib. Plasma Phys. 52, No. 5-6, 505 (2012).

[8] Y. Homma and A. Hatayama, J. Comput. Phys. 250, 206 (2013).

[9] S.I. Braginskii, Transport Processes in a Plasma, in Reviews of Plasma Physics, Vol.1 (M.A. Leontovich Ed.), (Consultants Bureau, New York, 1965).

[10] P. Helander and D.J. Sigmar, Collisional Transport in Magnetized Plasmas (Cambridge University Press, New York, 2002).

[11] T. Takizuka and H. Abe, J. Comput. Phys. 25, 205 (1977).

[12] B.A. Trubnikov, Particle Interactions in a Fully Ionized Plasma, in Reviews of Plasma Physics, Vol.1 (M.A. Leontovich Ed.) (Consultants Bureau, New York, 1965). 